\title{
Emergency Treatment on Gartland Type III Pediatric Supracondylar Humeral Fractures: a Retrospective Analysis of Therapeutic Consequences of Surgery
}

\section{Qian Wang}

Chengde Medical University Affiliated Hospital

\section{Yu Wang}

Chengde Medical University Affiliated Hospital

Jingxin Zhao ( $\sim$ zhaojingxin8459@sina.cn )

Chengde Medical University Affiliated Hospital https://orcid.org/0000-0003-1078-8236

\section{Research article}

Keywords: supracondylar humeral fracture, emergent reduction, open reduction, closed reduction

Posted Date: October 5th, 2021

DOI: https://doi.org/10.21203/rs.3.rs-948395/v1

License: (c) (i) This work is licensed under a Creative Commons Attribution 4.0 International License.

Read Full License 


\section{Abstract}

Objective: The objective of this analysis was to investigate the effect of emergency treatment by simple reduction within $8 \mathrm{~h}$ of injury for Gartland type III pediatric supracondylar humeral fractures.

Methods: One hundred twenty children with Gartland type III supracondylar humeral fractures were studied. All 120 patients had extension-type fractures and each was classified into one of two groups as follows: group $A(n=90)$, treated by closed reduction; group $B(n=30)$, treated by open reduction. Both groups underwent Kirschner wire internal fixation within 1 to $5 \mathrm{~d}$ after reduction. Information on the emergency treatments within $8 \mathrm{~h}$ of the fracture and the duration of the subsequent surgeries was collected and compared between the two groups.

Results: There was no significant difference between the two patient groups in terms of the demographic parameters. Compared to non-emergency surgery, emergency surgery required shorter operation time and fewer complications $(P<0.05)$. Nevertheless, the fracture healing time was significantly less $(P \llbracket 0.05)$, and Flynn scores were higher in the closed reduction group compared to the open reduction group $(P<0.05)$.Sixty-six of the 90 patients in group A received emergency treatment with an average subsequent surgery duration of $40 \mathrm{~min}$. The remaining 24 patients did not receive emergency treatment and had an average surgery duration of $65 \mathrm{~min}$. Of the 30 patients in group B, four received emergency treatment with a subsequent average surgery duration of $70 \mathrm{~min}$. The remaining 26 patients did not receive emergency treatment and had an average surgery duration of $91 \mathrm{~min}$.

Conclusion: Emergency treatment by simple reduction within $8 \mathrm{~h}$ of fracture was important for subsequent surgical procedures and duration. Lack of this treatment could increase the risks during open reduction. Emergency treatment within $8 \mathrm{~h}$ of fracture could increase the healing rate and reduce the duration of the subsequent surgery.

\section{Introduction}

Pediatric supracondylar humeral fracture is one of the most common fractures in children. Therapeutic outcomes have improved in recent years, and closed reduction with Kirschner wire percutaneous internal fixation, with improved fixing stability and less complications, is one of the best and broadly used treatments throughout clinics. Open reduction usually results in delayed healing, more surgical trauma, and more complications, which, although not favorable in most cases, is still used when closed reduction is difficult. Most studies have focused on the therapeutic effects between open and closed reduction surgery instead of on the factors that influence the final therapeutic outcomes. In the current study, treatment information on 120 patients with Gartland type III pediatric supracondylar humeral fractures was retrieved to investigate the significance of emergency treatment within $8 \mathrm{~h}$ of fracture, and to determine whether the lack of emergency treatment could increase the risks during subsequent open surgery. 


\section{Subjects And Methods}

\section{Patients}

One hundred twenty patients from the Chengde area of Hebei Province, China, and who had closed fractures of the extension type were studied. The inclusion criteria were as follows: patients aged between 2 and 12 years with Gartland type III supracondylar humeral fractures admitted in our hospital between January 2015 and May 2018. The 120 patients comprised 52 boys and 68 girls.

The exclusion criteria were as follows: open fracture, Gartland type II/I of supracondylar humeral fractures, or fractures with blood vessel injury in need of open surgery exploration.

\section{Emergency treatment}

Two kinds of treatment procedures were applied to the patients as follows: 1) the fracture was treated within $8 \mathrm{~h}$ with a simple reduction manipulation by the on-duty resident using a plaster fixation under the elbow flexion of $30^{\circ}$; the reduction was performed under traction until the bone friction could be tangible; soft tissue entrapment was avoided; 2 ) the fracture $>8 \mathrm{~h}$ before admission to the hospital with severe swelling was fixed only with plaster under the elbow flexion of $30^{\circ}$; there was reduction.

\section{Surgical treatment}

All surgeries were performed by the same surgeon who had 8 years' experience in pediatric orthopedics. The patients were placed in a prone or supine position according to the preference of the surgeon and were under general or brachial plexus anesthesia. The condition of the emergency treatment fracture reduction was observed anteroposterior under the elbow joint and by lateral X-ray. First, the proximal fracture was held by the surgeon's left hand, and the reduction was performed again by holding the internal and external condyle of the humerus with the right hand thumb and index finger, respectively. Then, the surgeon held the proximal fracture with both hands and used both thumbs to press against the ulna olecranon fossa for anteroposterior reduction. Three Kirschner wires with diameters ranging from 1.2 to $2.0 \mathrm{~mm}$ were inserted from the lateral side in the shape of the sector after reduction. If the closed reduction failed, a $2.0-\mathrm{cm}$ incision was made from the lateral side on the affected elbow to expose the proximal fracture followed by the open reduction. Similarly, three Kirschner wires with diameters ranging from 1.2 to $2.0 \mathrm{~mm}$ were inserted from the lateral side in the shape of the sector. The incision was closed layer by layer. Finally, a long anterior plaster fixation was applied with an elbow flexion of $100^{\circ}$ to remain for 3 to 4 weeks.

Four weeks after surgery, an X-ray of the elbow was taken, the plaster fixation and Kirschner wires were dissembled and removed, and elbow rehabilitation protocols were initiated. The elbow was reexamined every 2 months.

\section{Postoperative followup and evaluation}


All patients were followed up postoperatively for from 12 to 20 months (average, 14.5 months). Information on the emergency treatments before surgery were retrieved from medical records, and the surgery time was statistically analyzed. The function of the elbow was evaluated according to the elbow functional assessment scale (Flynn's scale). A flexion limitation $<5.0^{\circ}$ and a carrying angle between 10 and $15^{\circ}$ was considered excelent a flexion limitation $<5.0^{\circ}$ and a carrying angle between 5.0 and $9.0^{\circ}$ was considered good, a flexion limitation $<15^{\circ}$ and a carrying angle between 0 and $4.0^{\circ}$ was considered fair, and a flexion limitation $<15^{\circ}$ cubitus varus was considered poor.

\section{Statistical analyses}

The data were analyzed by SPSS 22.0 software. Descriptive statistics including means and frequencies were calculated for each of the examined variables. The treatment outcomes of the two surgical methods were compared using Independent-Sample t-test for Metrological data or chi square test for the counting dataas appropriate. $P<0.05$ was considered statistically significant.

\section{Results}

There were 90 patients in group A who underwent closed reduction with Kirschner wire percutaneous internal fixations, 66 of whom received emergency treatment within $8 \mathrm{~h}$ of the fracture. The duration of the subsequent for surgery for these 66 patients averaged $40 \mathrm{~min}$. The remaining 24 patients who did not receive emergency treatment had an average surgery duration of $60 \mathrm{~min}$. There were 30 patients in group $B$ who underwent open reduction with Kirschner wire percutaneous internal fixations, four of whom received emergency treatment within $8 \mathrm{~h}$ of fracture. The duration of the subsequent surgery for these patients averaged $70 \mathrm{~min}$. The remaining 26 patients who did not receive emergency treatment had an average surgery duration of $91 \mathrm{~min}$. For those patients receiving emergency simple reduction treatment within $8 \mathrm{~h}$ of the fracture, the fracture closure rate was $94.3 \%$; without emergency simple reduction treatment within $8 \mathrm{~h}$, the fracture closure rate was $48.0 \%$. The surgery duration was less in patients who had emergency simple reduction treatment than in those without emergency treatment, regardless of whether the surgery per se was an open or closed format.

In group A, the recovered elbow function was rated as good, fine, fair, and poor in 82, 7, 1, and 0 patient(s), respectively, and a Kirschner wire channel infection was found in two patients. In group $B$, the recovered elbow function was rated as good, fine, fair, and poor in 22, 6, 1, and 1 patient(s), respectively; incision infection and myositis ossificans were found in two patients. Our findings indicated that closed reduction was superior to open reduction for the treatment of supracondylar humeral fractures.

\section{Discussion}

Pediatric supracondylar humeral fracture is usually caused by hyperextension of the elbow joint, and Gartland type III factures were reported to occurred more frequently in summer[1] caused by injuries associated with high-energy activities, with severe damage to the surrounding soft tissue[2] associated 
with vascular and nerve injury.[3, 4] All Gartland type III fractures required reduction and fixation $[5,6]$ using different approaches, such as open reduction, closed reduction, or external fixation.[7, 8, 9] Complications were usually associated with open reduction ; therefore, closed reduction with Kirschner wire percutaneous internal fixation was generally accepted as the optimal approach.[10-14].

The supracondylar humeral fracture is a complete displaced fracture. Given the thin cortex of the supracondylar bone, reduction is usually difficult, although most cases could be managed by closed reduction with Kirschner wire percutaneous internal fixation. Because intraoperative reduction might be repeated, a thorough and adequate preoperative preparation is critical to avoid surgical injuries to nerves, blood vessels, and soft tissues. It was suggested that there was no significant difference between emergency surgery and delayed surgery for the treatment of pediatric supracondylar humeral fractures[15-18]; however, emergency treatment at the early stages of a fracture was crucial, and timely reduction and fixation were very important once the patient was admitted. At the early stages of fracture, swelling of the affected limb is not yet at its severest, and the fracture ends are still touchable in most cases. A simple reduction is conducted with the objective of connecting the two fracture ends without soft tissue incarcerated. In the other words, the aim is to convert a Gartland type III fracture to a Gartland type II fracture. The advantages of emergency treatment on Gartland type III fractures are summarized according to the following information received from our retrieved medical records: 1) emergency treatment significantly reduced surgery duration compared to no emergency treatment with high closure rates and less complications; 2) Gartland type III fractures were usually associated with nerve or blood vessel injuries, the most common of which was brachial artery spasms or entrapment; emergency simple reduction treatment could improve blood supply in the hands and release the stimuli on the nerves to promote recovery; 3 ) only minor reduction or adjustment was needed during the subsequent surgery, and repeated intraoperative traction and secondary injuries on surrounding tissue could be avoided; 4) emergency reduction treatment and long anterior plaster fixation with an elbow flexion at $30^{\circ}$ could reduce pain and swelling by enabling hand activity; 5) the body surface marker was overt at the early stages of fracture with less extension of surrounding tissues, which benefited reduction manipulations; 6 ) our respective findings indicated that there were multiple reasons behind the failure of closed reduction with Kirschner wire percutaneous internal fixation, $[19,20]$ including the intraoperative reduction method, experience of the surgeon, and timing of the surgery. Emergency reduction on the fracture in the early stages was one of the most important factors for the success of closed reduction with Kirschner wire percutaneous internal fixation for the treatment of Gartland type III supracondylar humeral fractures.

\section{Abbreviations}

CA: carrying of angle

\section{Declarations}

- Ethics approval and consent to participate 
Ethics approval and consent to participate in present study was approved by the Ethics Committee of Affiliated Hospital of Chengde Medical College

- Consent for publication

Not applicable.

- Availability of data and materials

All data generated or analyzed during this study are included in this manuscript.

- Competing interests

The authors declare that they have no competing interests.

- Funding

No funding was provided.

- Authors' contributions

JZ contributed to the study design and is the corresponding author. QW and JZ contributed to the study design, data analysis and interpretation, and manuscript draft.QW and YW contributed to the data collection and analysis. YW and JZ contributed to the literature search and manuscript revision. All authors have read and approved the final manuscript.

- Acknowledgements

We thank all of the patients involved in the study

\section{References}

1. Barr LV. Paediatric supracondylar humeral fractures: epidemiology, mechanisms and incidence during school holidays. J Child Orthop. 2014 Mar;8(2):167-70.

2. Sun LJ, Wu ZP, Yang J.etal. Factors associated with a failed closed reduction for supracondylar fractures in children. Orthop Traumatol SurgRes .2014 Oct;100(6):621-4.

3. Saglam Y, Tunali O, Akgul T, et al. Mid-term results of pediatric vascular injured supracondylar humerus fractures and surgical approach. J Pediatr Orthop B. 2014 Nov;23(6):572-8.

4. Joiner ER1, Skaggs DL, Arkader A, et al. latrogenic nerve injuries in the treatment of supracondylar humerus fractures: are we really just missing nerve injuries on preoperative examination? J Pediatr Orthop. $2014 ; 34(4): 388-92$.

5. Mohammed R, Bhogadi P, Metikala S. A study of biplanar crossed pin construct in the management of displaced. pediatric supracondylar humeralfractures J Child Orthop. 2014 Sep 3 
6. Marashi Nejad SA, Mehdi Nasab SA, Baianfar M2. Effect of supination versus pronation in the nonoperative treatment of pediatric supracondylar humerusfractures. Arch Trauma Res. 2013 Spring;2(1):26-9.

7. Hussain S, Dhar S, Qayoom A. Open Reduction and Internal Fixation of displaced Supracondylar Fractures of Humerus with Crossed K-wires via Medial Approach. Malays Orthop [J]. 2014 Jul;8(2):29-34.

8. Hussain S1, Ahmad M, Muzaffar T. Open reduction and internal fixation for displaced supracondylar fractures of the humerus in children with crossed K-wires via lateral approach. Chin J Traumatol. 2014 Jun 1;17(3):130-5.

9. Slongo T.Radial external fixator for closed treatment of type III and IV supracondylar humerus fractures in children. A new surgical technique[Oper Orthop Traumatol. 2014 Feb;26(1):75-96

10. Wu LG, Yang SB. Closed reduction and fixation for the treatment of Gartland type II and III supracondylar fracture of humerus in children[Zhongguo Gu Shang. 2013 Feb;26(2):98-101.

11. Pennock AT1, Charles M, Moor M, et al. Potential causes of loss of reduction in supracondylar humerus fractures. J Pediatr Orthop. $2014 ; 34(7): 691-7$.

12. Sahu RL.Percutaneous K-wire fixation in paediatric Supracondylar fractures of humerus: A retrospective study. Niger Med J. $2013 ; 54(5): 329-34$.

13. Jakob LV1, Acklin YP, Furrer M, et al. Pediatric supracondylar fractures of the humerus Praxis . 2013 ;102(5):285-91.

14. Herzog MA1, Oliver SM, Ringler JR, et al. Mid-America Orthopaedic Association Physician in Training Award: Surgical technique: Pediatric supracondylarhumerus fractures: a technique to aid closed reduction. Clin Orthop Relat Res. 2013 ;471(5):1419-26.

15. Novais EN, Andrade MA, Gomes DC.The use of a joystick technique facilitates closed reduction and percutaneous fixation of multidirectionally unstable supracondylar humeral fractures in children. $J$ Pediatr Orthop. $2013 ; 33(1): 14-9$.

16. Kronner JM Jr1, Legakis JE1, Kovacevic N1, et al.An evaluation of supracondylar humerus fractures: is there a correlation between postponing treatment and the need for open surgical intervention? $\square \mathrm{J}$ Child Orthop. 2013 Mar;7(2):131-7.

17. Larson AN1, Garg S, Weller A, et al. Operative treatment of type II supracondylar humerus fractures: does time to surgery affect complications? J Pediatr Orthop. 2014 ;34(4):382-7.

18. Garg S1, Weller A, Larson AN, et al. Clinical characteristics of severe supracondylar humerus fractures in children. J Pediatr Orthop. $2014 ; 34(1): 34-9$.

19. Holgado Moreno E1, Tomé-Bermejo F, Ruiz Micó N. Open reduction and internal fixation of supracondylar fractures of the humerus in children. Analysis of results at ten-year of follow-up $\square$ Rev Esp Cir Ortop Traumatol. 2012; 56(5):361-8.

20. Beck JD1, Riehl JT, Moore BE, et al. Risk factors for failed closed reduction of pediatric supracondylar humerus fractures. Orthopedics. $2012 ; 35(10):$ e1492-6. 


\section{Tables}

Table 1. basic condition of patients

\begin{tabular}{|c|c|c|c|}
\hline Group & Closed reduction & Open reduction & $P$ \\
\hline Gender ( male / female)* & $40: 50$ & $12: 18$ & 0.671 \\
\hline Age (years old) & $6.39 \pm 2.66$ & $6.80 \pm 2.76$ & 0.980 \\
\hline Height(cm) & $119.23 \pm 15.73$ & $124.33 \pm 17.99$ & 0.162 \\
\hline Weight(kg) & $25.74 \pm 9.89$ & $31.29 \pm 11.77$ & 0.161 \\
\hline Affected side (example, left / right)* & $48: 42$ & $22: 8$ & 0.054 \\
\hline Operation time(min) & $45.33 \pm 17.17$ & $88.20 \pm 9.73$ & $\triangle 0.001$ \\
\hline Emergency treatment* & $66: 24$ & $4: 26$ & $\triangle 0.001$ \\
\hline Mean operating time(min) & 40 & 70 & \\
\hline
\end{tabular}

*Use the chi-square test to calculate the $\boldsymbol{P}$ value

Table 2. Comparison of Flynn scores of elbow joint in children 12 months after operation

\begin{tabular}{|llll|}
\hline Flynn score & Closed reduction & Open reduction & $\boldsymbol{p}$ \\
\hline According to CA* & & & 0.033 \\
\hline Excelent & $82(91.1 \%)$ & $22(73.3 \%)$ & \\
\hline Good & $7(7.8 \%)$ & $6(20 \%)$ & \\
\hline Fair & $1(1.1 \%)$ & $1(3.3 \%)$ & \\
\hline Poor & 0 & $1(3.3)$ & \\
\hline
\end{tabular}

*Use the chi-square test to calculate the $\boldsymbol{P}$ value

Figures 

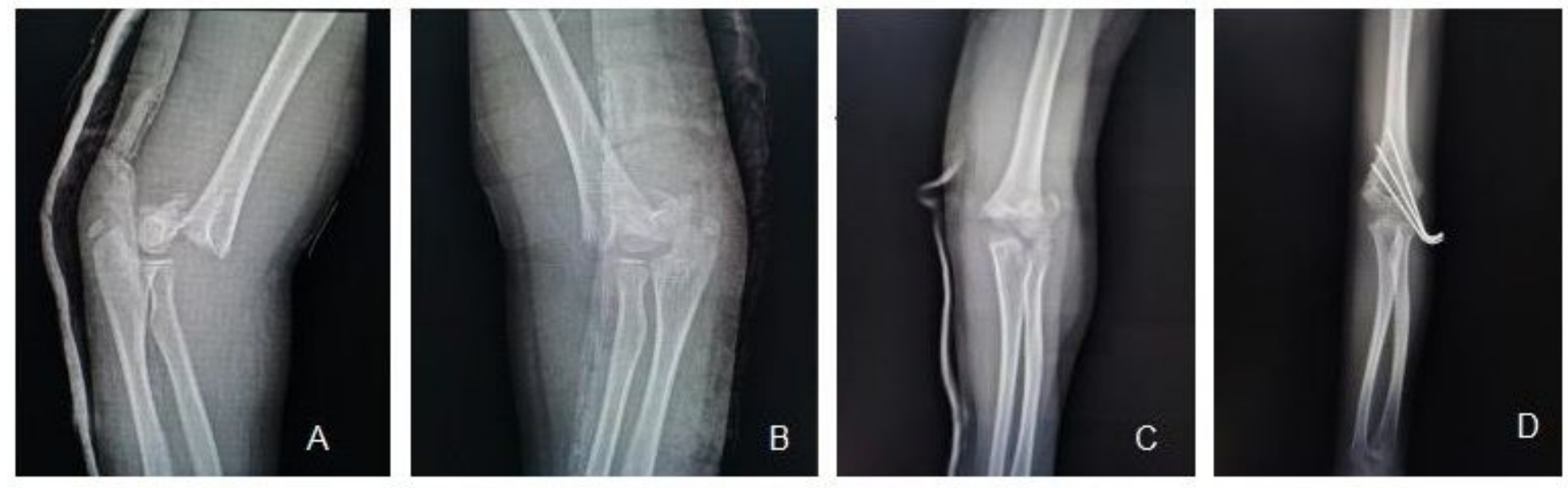

Figure 1

$[A \square B]$ : X-ray of the preoperative supracondylar fracture of humerus [C$\square \mathrm{D}]$ : $\mathrm{X}$-ray of the postoperative supracondylar fracture of humerus 